data were retrieved from Danish population-based registers. Incremental cost-effectiveness rates (ICERs) were calculated on the basis of a comparison of the development in costs and effects in the two intervention groups (separately and combined) with the control group. Bootstrap with 10,000 replications were used to access significance.

Results: The difference in health and social care costs during the intervention period compared to the year before were $€ 1,072$, - $€ 50$ and $€ 519$ for the control group, the PRO-TR group and the PRO-TN respectively. Hence, the change in health and social care costs was lower for both intervention groups. The PRO-TR group had a small decrease and it was significantly lower than for the control group $(p=0.0027)$. The difference between health and social care costs in the PRO-TN group compared to the control group was only borderline significant $(p=0.067)$. No statistically significant differences were found in QALY's between the three groups, all three groups experienced minor, non-significant, declines in QALY over the intervention period. ICER's were not statistically significant but below known threshold values for the PRO-RN group (ICER=€17,121)

Conclusion: It is difficult to obtain statistically significant results for cost-effectiveness in small samples. However, the results point towards a possible cost-saving impact of PRO interventions in patients with low disease activity or remission. The study was unable to conclude if PRO-TR or PRO-TN were most cost-effective. Other relevant considerations, like patient satisfaction or organisational issues, should determine the way of organizing RA disease management in these patients.

References:

[1] de Thurah A, Stengaard-Pedersen K, Axelsen M, et al. Tele-Health Follow-up Strategy for Tight Control of Disease Activity in Rheumatoid Arthritis: Results of a Randomized Controlled Trial. Arthritis care \& research 2018; 70(3): 353-60.

Disclosure of Interests: Annette de Thurah Grant/research support from: Novartis (not relevant for the present study)., Speakers bureau: Lily (not relevant for the present study)., Christian Skovsgaard: None declared, Thomas Maribo: None declared, Niels Henrik Hjøllund: None declared, Marie Kruse: None declared

DOI: 10.1136/annrheumdis-2020-eular.4449

\section{OP0157-HPR DISSEMINATION AND ASSESSING IMPLEMENTATION OF THE EULAR RECOMMENDATIONS FOR PATIENT EDUCATION FOR PEOPLE WITH INFLAMMATORY ARTHRITIS ACROSS EUROPE}

S. Bennett ${ }^{1}$, H. A. Zangi ${ }^{2}$, A. Van Tubergen ${ }^{3}$, M. Ndosi ${ }^{1}$ on behalf of STOPE Collaborators. ${ }^{1}$ University of the West of England - UWE Bristol, Stoke Gifford, United Kingdom; ${ }^{2}$ Diakonhjemmet Hospital, Oslo, Norway; ${ }^{3}$ Maastricht University, Maastricht, Netherlands

Background: EULAR recommendations for patient education for people with inflammatory arthritis (IA) were published in $2015 .{ }^{1}$ This is the first systematic dissemination and implementation study, reporting qualitative data identifying barriers to implementation of the recommendations.

Objectives: To (i) disseminate and assess the level of acceptability and applicability of the EULAR recommendations for patient education among healthcare professionals and rheumatologists and (ii) assess potential barriers and facilitators to their application in clinical practice.

Methods: This was a cross-sectional study using survey methods. Survey items were developed in English and translated into 20 different languages before being distributed to health professionals by snowball sampling using an online platform. The items covered demographic information and 10-point rating scales assessing the level of acceptability and applicability of each of the eight recommendations. In addition, textual data fields were provided to assess reasons for disagreeing and barriers to implementation of the recommendations in practice. Quantitative data were analysed descriptively with agreement and applicability levels summarised as median (IQR) scores. Textual data were analysed by content analysis and presented in themes. Finally, collaborators in each country reviewed the top barriers to implementation and proposed facilitators to implementation in their respective countries.

Results: A total of 2442 responses were recorded from 23 countries, but only 1495 contained complete data. Of complete responses, $74 \%$ were women. Most of the professionals were nurses $(n=640)$, rheumatologists $(n=369)$ and physiotherapists $(n=232)$

Table 1 presents the levels of agreement and applicability of the recommendations. For all recommendations, the level of agreement was high (median=10). However, the level of applicability was generally lower compared to each corresponding agreement level, especially for recommendation 6 , which states that the effectiveness of patient education should be evaluated. Lack of an effective evaluation tool was the biggest barrier to implementation.

Table 1. Levels of agreement and applicability of each recommendation.

\begin{tabular}{lccccc}
\hline & \multicolumn{2}{c}{ Agreement } & & \multicolumn{2}{c}{ Applicability } \\
\cline { 2 - 3 } & Median & IQR & & Median & IQR \\
\hline Recommendation 1 & 10 & 10 to 10 & & 8 & 7 to 10 \\
Recommendation 2 & 10 & 10 to 10 & & 8 & 6 to 10 \\
Recommendation 3 & 10 & 9 to 10 & & 8 & 7 to 10 \\
Recommendation 4 & 10 & 8 to 10 & & 7 & 5 to 10 \\
Recommendation 5 & 10 & 8 to 10 & & 7 & 5 to 9 \\
Recommendation 6 & 10 & 8 to 10 & & 6 & 4 to 8 \\
Recommendation 7 & 10 & 9 to 10 & & 8 & 5 to 8 \\
Recommendation 8 & 10 & 10 to 10 & 8 & 5 to 8 \\
\hline
\end{tabular}

There were notable similarities between barriers and facilitators for implementation of the recommendations across countries. The 3 most common barriers to application were; (i) lack of time (ii) lack of training in how to provide patient education and (iii) not having enough staff to provide patient education. The most common facilitators were: tailoring the content and delivery of patient education to individual patients; training providers, and evaluating the effectiveness of patient education with individual patients.

Conclusion: This project has disseminated the EULAR recommendations for patient education across 23 countries. There was high agreement with the recommendations among health professionals but their application to clinical practice was lower. Some barriers to application are amenable to change such as addressing training needs of health professionals and developing evaluation tools for patient education.

1. Patient education should be provided for people with inflammatory arthritis as an integral part of standard care in order to increase patient involvement in disease management and health promotion.

2. All people with inflammatory arthritis should have access to and be offered patient education throughout the course of their disease including as a minimum; at diagnosis, at pharmacological treatment change and when required by the patient's physical or psychological condition.

3. The content and delivery of patient education should be individually tailored and needs-based for people with inflammatory arthritis.

4. Patient education in inflammatory arthritis should include individual and/or group sessions, which can be provided through face-to-face or online interactions, and supplemented by phone calls, written or multimedia material.

5. Patient education programmes in inflammatory arthritis should have a theoretical framework and be evidence-based, such as selfmanagement, cognitive behavioural therapy or stress management.

6. The effectiveness of patient education in inflammatory arthritis should be evaluated and outcomes used must reflect the objectives of the patient education programme.

7. Patient education in inflammatory arthritis should be delivered by competent health professionals and/or by trained patients, if appropriate, in a multidisciplinary team.

8. Providers of patient education in inflammatory arthritis should have access to, and undertake, specific training in order to obtain and maintain knowledge and skills.

Figure 1. Recommendations for patient education in inflammatory arthritis. ${ }^{1}$

\section{References:}

[1] Zangi HA, et al. EULAR recommendations for patient education for people with inflammatory arthritis Ann Rheum Dis. 2015;74:954-962.

Disclosure of Interests: Sarah Bennett: None declared, Heidi A. Zangi: None declared, Astrid van Tubergen Consultant of: Novartis, Mwidimi Ndosi Grant/ research support from: Bristol Myers Squibb, Consultant of: Janssen, Pfizer DOI: 10.1136/annrheumdis-2020-eular.3251 